Ana Carolina Ayeta ${ }^{1}$

ana Cristina Barros da CUnha ${ }^{2}$

Sonaly Petronilho Heidelmann ${ }^{3}$

Cláudia SAUNDers ${ }^{4}$

Artigo Original

Palavras-chave

Pica

Gestação

Estresse psicológico

Ansiedade

Transtornos nutricionais

Keywords

Pica

Pregnancy

Stress, psychological

Anxiety

Nutrition disorders

\title{
Fatores nutricionais e psicológicos associados com a ocorrência de picamalácia em gestantes
}

\author{
Nutritional and psychological factors associated \\ with the occurrence of pica in pregnant women
}

\section{Resumo}

OBJETIVO: Avaliar fatores nutricionais e psicológicos associados com a ocorrência da prática da picamalácia em gestantes atendidas em maternidade pública do Rio de Janeiro, Brasil. MÉTODOS: Estudo descritivo, de caráter exploratório, realizado com 13 gestantes (idades entre 16 e 40 anos) com diagnóstico de picamalácia na gestação atual identificada em entrevista padronizada na consulta com nutricionista que abordava questões sobre ocorrência e frequência do transtorno, além dos tipos de substâncias ingeridas. Após assinarem o Termo de Consentimento Livre e Esclarecido (TCLE), todas as gestantes passaram por avaliação nutricional e, posteriormente, sete foram submetidas à avaliação psicológica com instrumentos padronizados para medida de ansiedade e estresse, além da avaliação de estratégias de enfrentamento (coping) diante de estressores. RESULTADOS: As práticas de picamalácia mais referidas foram pagofagia $(30,8 \%)$ e fruta com sal $(30,8 \%)$. Observou-se ocorrência de algum grau de estresse e ansiedade em todas as gestantes com picamalácia, assim como alguma comorbidade $(69,2 \%)$ e sintomatologia digestiva $(84,6 \%)$. As estratégias de coping mais prevalentes foram "focada em práticas religiosas/pensamento fantasioso" e "busca de suporte social", ambas com 42\%. CONCLUSÃO: Considerando que a picamalácia pode associar-se com maior risco perinatal, é fundamental que essa prática seja investigada durante o atendimento pré-natal e que ocorra a adoção de práticas preventivas obstétricas, psicológicas e nutricionais para reduzir complicações para a mãe e o concepto.

\section{Abstract}

PURPOSE: To evaluate the nutritional and psychological factors associated with the occurrence of the practice of pica in pregnant women attending a public hospital in Rio de Janeiro, Brazil. METHODS: The study was based on a descriptive design with exploratory features, and conducted on 13 adult and adolescent pregnant women aged 16 to 40 years with a diagnosis of pica in the current pregnancy. Pica was diagnosed by a nutritionist in a standardized interview situation, when questions about the occurrence and frequency of pica, and types of substance ingestion were investigated. After signing the Informed Consent Form (ICF), all participants were evaluated by a nutritionist and seven of them were submitted to psychological assessment with standardized instruments to evaluate stress and anxiety, and to assess coping strategies. RESULTS: The type of pica most frequently reported was pagophagia $(30.8 \%)$ and the consumption of fruit with salt (30.8\%). The most prevalent coping strategies were "religious practice-focused" and "seeking social support", both presented by $42 \%$ of the pregnant women. We observed the occurrence of some degree of stress and anxiety in all pregnant women, as well as comorbidities (69.2\%) and gastrointestinal symptoms (84.6\%). CONCLUSION: Considering that pica may be associated with increased perinatal risk, it is very important to investigate this disorder during prenatal care, and to dopt obstetric, psychological and nutritional preventive practices to reduce the complications for mother and fetus.

Correspondência

Cláudia Saunders

Departamento de Nutriç̄õo e Dietética. Instituto de Nutricão Losué de Castro. Universidade Federal do Rio de Janeiro Avenida Carlos Chagas Fihho, 373, bloco J, $2^{\circ}$ andar, sala 26 -

Cidade Universitárí

CEP: 21941-590

Rio de Janeiro (RJ), Brasil

Recebido

$16 / 06 / 2015$
Ambulatório de Pré-natal da Maternidade Escola da Universidade Federal do Rio de Janeiro - UFRJ - Rio de Janeiro (RJ), Brasil. IPrograma de Residência Multiprofissional em Saúde Perinatal, Maternidade Escola, Universidade Federal do Rio de Janeiro - UFRJ Rio de Janeiro (RJ), Brasil.

2Instituto de Psicologia, Universidade Federal do Rio de Janeiro - UFRJ; Maternidade Escola, Universidade Federal do Rio de Janeiro UFR - Rio de Janeiro (RJ), Brasil.

${ }^{3}$ Instituto de Nutrição Josué de Castro, Universidade Federal do Rio de Janeiro - UFRJ; Maternidade Escola, Universidade Federal do Rio de Janeiro - UFRJ - Rio de Janeiro (RJ), Brasil.

${ }^{4}$ Instituto de Nutrição Josué de Castro, Universidade Federal do Rio de Janeiro - UFRJ: Maternidade Escola, Universidade Federal do Rio de Janeiro - UFRJ; Bolsista PQ/CNPq - Rio de Janeiro (RJ), Brasil.

Conflito de interesses: não há. 


\section{Introdução}

Diferentes termos são utilizados para descrever a desordem alimentar conhecida como picamalácia, caracterizada pela ingestão persistente e compulsiva de substâncias inadequadas, alimentos esdrúxulos, condimentos raros ou substâncias estranhas, com pequeno ou nenhum valor nutritivo, ou de substâncias comestíveis, mas não na sua forma habitual (combinações que normalmente não fazem parte dos hábitos alimentares). Outros termos adotados são: pica, picacia, picacismo, malácia, geomania, pseudorexia, entre outros ${ }^{1-6}$.

Segundo a Associação Americana de Psiquiatria (DSM-IV) ${ }^{7}$, a picamalácia é a ingestão persistente de substâncias não nutritivas por período de pelo menos um mês; ou a ingestão de substâncias não nutritivas impróprias para saúde; ou um comportamento alimentar que não faz parte da cultura praticada; ou um comportamento alimentar que ocorre exclusivamente durante o curso de um transtorno mental. Assim, o diagnóstico diferencial de picamalácia deve ser feito se o comportamento alimentar é suficientemente severo para indicar atenção clínica independente ${ }^{7}$.

As práticas de picamalácia mais comuns na gestação são pagofagia (ingestão excessiva de gelo), geofagia (ingestão de terra/barro), amilofagia (ingestão de goma, principalmente de lavanderia), consumo de miscelâneas (combinações atípicas) e de frutas verdes. Outras substâncias não alimentares também são referidas, como palitos de fósforo queimados, cabelo, pedra e cascalho, carvão, fuligem, cinzas, comprimidos de antiácidos, leite de magnésia, borra de café, bolinhas de naftalina, pedaços de câmara de ar, plástico, tinta, sabonete, giz, toalha de papel e, até mesmo, sujeira ${ }^{1,2,4,8,9}$.

A etiologia da picamalácia parece ser complexa. Revisão de literatura sobre transtornos alimentares na gravidez indica que diversos fatores (ambientais, nutricionais, socioeconômicos, fisiológicos, culturais e psiquiátricos) têm sido reconhecidos como causa da picamalácia e de outros transtornos ${ }^{10}$. Além disso, fatores emocionais parecem determinar a associação entre alterações hormonais e estresse $\mathrm{e}^{5,10}$, sendo tais alterações responsáveis, inclusive, por ocasionar mudanças orgânicas e comportamentais significativas, com desencadeamento ou exacerbação de sintomatologia depressiva, ansiedade, baixa concentração, irritabilidade, mudança no apetite, insônia, hipersônia e perda de energia ${ }^{11}$.

A ansiedade é entendida como um componente emocional que acompanha todo o período gestacional até o puerpério, sendo caracterizada por estados de insatisfação, intranquilidade, insegurança, incerteza e medo da experiência desconhecida ${ }^{12}$. Sentimentos ambivalentes estão presentes desde o primeiro trimestre, variando de um medo inicial do aborto, oscilações de humor, desconfortos como náuseas, sonolência e cansaço até uma ansiedade com caráter de maior introversão e passividade, seguida de alterações do desejo e do desempenho sexual, da imagem corporal e da percepção dos movimentos fetais, quando o concepto já pode ser concretamente sentido ${ }^{12}$.

Esse estado emocional característico da gravidez é marcado por ansiedade e, por vezes, estresse, podendo resultar em comportamentos de enfrentamento (coping) pouco funcionais ou adaptativos, como é o caso da picamalácia. Nessa perspectiva, insere-se o conceito de coping, ação intencional, física ou mental, do indivíduo em resposta a um estressor percebido, dirigida para circunstâncias externas ou estados internos (medo, ansiedade, angústia) ${ }^{13}$. Compreendido como uma tentativa do organismo de se adaptar diante de uma situação de estresse, trata-se de uma estratégia aprendida, usada e adaptada para cada situação em particular, com base em um processo dinâmico pautado na percepção e interpretação do evento estressor, assim como em mecanismos de autorregulação moderados por características de temperamento e personalidade ${ }^{14}$. Respostas ou estratégias de enfrentamento (ou coping) podem focar-se no problema, na emoção ou reunir ambos os esforços, de acordo com sua função no manejo do estressor $^{14}$. Estudos nessa área ${ }^{14,15}$ afirmam existir diferentes tipos de estratégias de enfrentamento, como busca de suporte social/emocional, negação, aceitação, religiosidade, dentre outras, que podem atuar simultaneamente no enfrentamento de uma situação específica.

Indica-se como fundamental a avaliação de indicadores emocionais, como ansiedade e estresse, e das variáveis envolvidas no processo de enfrentamento de condições de risco, como a picamalácia. Tal avaliação pode subsidiar propostas de intervenção multiprofissional preventiva e promotoras de estratégias mais resilientes de enfrentamento em contextos de vulnerabilidade, principalmente quando se considera a picamalácia associada a desfechos indesejáveis na gravidez.

Intercorrências gestacionais podem associar-se à picamalácia, como as síndromes hipertensivas na gravidez, infecção do trato urinário, interferências na absorção de nutrientes, envenenamento por chumbo e hipercalemia ${ }^{16,17}$, além de anemia, constipação, distensão, obstrução intestinal, problemas dentários, infecções parasitárias, toxoplasmose, diabetes mellitus gestacional (DMG) ${ }^{2}$, e estudo recente sugere que a prática de picamalácia aumenta a chance de parto cirúrgico ${ }^{18}$. Além disso, as repercussões para o concepto incluem parto prematuro, baixo peso ao nascer, irritabilidade do neonato, perímetro cefálico fetal diminuído, exposição fetal a substâncias químicas, tais como chumbo, pesticidas e herbicidas, e, por fim, aumento do risco de morte perinatal ${ }^{8}$.

Importante considerar que a gravidez, que já é um momento de grandes expectativas e mudanças na vida da mulher, pode ser um período ainda mais estressante e propenso a problemas psicológicos e nutricionais quando ocorre sob condições clínicas como diabetes mellitus (DM), 
hipertensão arterial sistêmica, infecção do trato urinário e outras comorbidades. Nesses casos, é comum um estado emocional permeado de inseguranças, medos e angústias, que culminam em estresse significativo. Muitas vezes, para lidar com essa condição estressante, a mulher adota estratégias inadequadas para manejar essa instabilidade física e emocional. Nesse contexto pode ocorrer a picamalácia, desordem alimentar pouco estudada que pode se associar a resultado perinatal desfavorável ${ }^{1,2,5,19}$. Em face do exposto, objetivou-se avaliar os fatores nutricionais e psicológicos associados com a ocorrência da prática da picamalácia em gestantes atendidas em maternidade pública do Rio de Janeiro, assim como possíveis relações entre tais fatores e essa prática.

\section{Métodos}

Estudo descritivo, com caráter exploratório, que incluiu 13 gestantes com idades entre 16 e 40 anos (média de 28,1 anos) atendidas em maternidade pública do Rio de Janeiro, Brasil, entre julho e outubro de 2013. A pesquisa foi aprovada pelo Comitê de Ética em Pesquisa da Maternidade Escola da Universidade Federal do Rio de Janeiro (UFRJ), em 06 de setembro de 2012 (CAAE 06386412.1.0000.5275). Todas as participantes assinaram o Termo e Consentimento Livre e Esclarecido (TCLE).

Os critérios de inclusão da amostra adotados foram: gestantes de feto único, com início da assistência pré-natal até a $30^{a}$ semana gestacional e que referissem prática de picamalácia durante a gestação.

Todas as gestantes foram acompanhadas no pré-natal na unidade, incluindo pelo menos quatro consultas com o nutricionista e com o psicológico, com pelo menos uma consulta de avaliação psicodiagnóstica.

Realizou-se avaliação nutricional detalhada, incluindo avaliação dietética, antropométrica, clínica, bioquímica, funcional, sociodemográfica e obstétrica. Investigou-se a prática de picamalácia, ou seja, a ingestão de substâncias não alimentares e combinações atípicas, combinações de alimentos citadas pelas próprias gestantes e definidas por elas como estranhas e que não faziam parte do seu hábito alimentar. Para identificação dos casos de picamalácia, empregou-se a entrevista padronizada ${ }^{4,16}$ em todas as consultas com o nutricionista, que incluía questões sobre a ocorrência e a frequência do transtorno, além das substâncias ingeridas.

Para avaliação antropométrica, calculou-se o índice de massa corporal (IMC) pré-gestacional com base no peso informado ou medido até a $13^{\mathrm{a}}$ semana de gestação. $\mathrm{Na}$ avaliação das adultas consideraram-se os pontos de corte recomendados pelo Institute of Medicine (IOM) ${ }^{20}$; para as adolescentes, foram considerados os pontos de corte próprios para idade, segundo a recomendação do Ministério da Saúde adaptada ${ }^{21}$. A adequação do ganho de peso gestacional total foi avaliada segundo o $\mathrm{IOM}^{20}$.
$\mathrm{Na}$ avaliação clínica foram investigados a sintomatologia digestiva, os sinais sugestivos de carências nutricionais específicas e as intercorrências gestacionais, identificadas por meio de consulta aos prontuários e avaliação dos exames laboratoriais. Considerou-se anemia na gestação, concentrações de hemoglobina $<11 \mathrm{~g} / \mathrm{dL}^{22}$, por trimestre gestacional.

As condutas adotadas para as intercorrências gestacionais na unidade estudada se baseavam nas recomendações oficiais do Ministério da Saúde ${ }^{22}$, destacando-se a suplementação de ferro e ácido fólico para casos de anemia, além de orientação alimentar específica para cada intercorrência ${ }^{4,22}$.

As gestantes identificadas como praticantes de picamalácia na consulta com o nutricionista eram encaminhadas para avaliação psicodiagnóstica, realizada com a aplicação dos instrumentos padronizados: Inventário de Ansiedade Beck (BAI), com normas e padronização brasileiras ${ }^{23}$, usado para avaliar a ansiedade, classificando-a em níveis de gravidade crescentes, segundo os sinais e sintomas: mínimo (escore 1-7), leve (escore 8-15), moderado (escore 16-25) e grave (escore 26-63); Inventário de Sintomas de Stress para Adultos de Lipp ${ }^{24}$, padronizado para população brasileira, usado para avaliar sintomas de estresse, classificados em três tipos (somático/físico, psicológico ou mistos) e quatro fases:

1. alerta: fase conhecida como reação saudável ao estresse, na qual o indivíduo é capaz de lidar com o estímulo estressor e, em seguida, retornar ao seu equilíbrio;

2. resistência: fase caracterizada pela busca de homeostase do desequilíbrio gerado pela fase de alerta, quando o indivíduo, com maior desgaste emocional, fica suscetível a desenvolver algum quadro sintomatológico e manifestações psicossociais;

3. quase exaustão: fase caracterizada pelo enfraquecimento da pessoa, com consequente dificuldade de ajustamento e resistência à situação, mas capacidade preservada de executar algumas tarefas, apesar de grande cansaço;

4. exaustão: considerada a fase patológica do estresse, caracterizada pela permanência de estímulos estressantes que desencadeiam falhas na capacidade adaptativa do organismo, com importante desequilíbrio interior, ocorrência de depressão e produtividade nula.

A Escala Modos de Enfrentamento de Problemas $(\text { EMEP })^{25}$ foi usada para avaliação do coping com base em análise clínica ou qualitativa de estratégias de enfrentamento diante de estresse, classificadas em quatro tipos:

1. focalizadas no problema;

2. focalizadas na emoção;

3. focalizadas em práticas religiosas/pensamento fantasioso;

4. focalizadas na busca de suporte social.

$\mathrm{Na}$ análise da EMEP efetua-se o somatório dos valores assinalados, em que a maior mediana dos escores indica a estratégia de enfrentamento mais prevalente. 
Toda a coleta de dados foi feita por pesquisadores treinados e supervisionados, com uniformização dos procedimentos seguindo consulta aos prontuários, aos registros da equipe de saúde, assim como entrevistas individuais com as gestantes, com preenchimento de protocolos e instrumentos de avaliação padronizados e pré-testados. Os dados foram analisados de acordo com as normas dos instrumentos de avaliação psicológica. Foram estimadas as medidas de tendência central - média e desvio padrão (DP) - com o auxílio do programa Statistical Package for the Social Sciences (SPSS), versão 21.

\section{Resultados}

Do total de participantes, três eram adolescentes, e dez, adultas, que compareceram, em média, por dez consultas de atendimento pré-natal. A maioria das gestantes $(69,2 \% ; \mathrm{n}=9)$ tinha o ensino médio completo, era dona de casa $(53,8 \%$; $\mathrm{n}=7$ ) e exercia trabalho remunerado como doméstica, auxiliar de escritório ou técnica de enfermagem $(46,1 \% ; n=6)$.

Quanto às características antropométricas, verificou-se que $53,8 \%$ das gestantes apresentaram IMC pré-gestacional normal, 23,1\% de sobrepeso e 23,1\% de obesidade. Verificou-se que $69,2 \%$ das gestantes apresentavam algum tipo de comorbidade, como diabetes mellitus tipo 2 - DM2 $(22,2 \% ; n=2) ; \mathrm{DMG}(66,6 \%$; $\mathrm{n}=6)$; infecção do trato urinário $(11,1 \% ; \mathrm{n}=1)$; hipertensão gestacional $(11,1 \% ; \mathrm{n}=1)$; hipotireoidismo $(11,1 \% ; \mathrm{n}=01)$. Observou-se ainda que $84,6 \%(n=11)$ delas apresentavam pirose, enjoo, êmese e constipação intestinal. Uma gestante $(7,7 \%)$ que praticava a picamalácia apresentou anemia no $3^{\circ}$ trimestre gestacional.

Observaram-se diferentes tipos de prática de picamalácia, sendo que algumas gestantes relataram polipicamalácia naquela gestação, como, por exemplo, consumo excessivo de gelo simultâneo ao consumo de combinação atípica de alimentos. Outras gestantes referiram práticas diferentes de picamalácia: consumiam "terra molhada" no primeiro trimestre e passaram a consumir "maçã com ketchup" no segundo trimestre gestacional. Combinações bastante exóticas foram identificadas, como "peixe frito com geleia de morango", "sorvete com tomate" e "frango com leite condensado", todos com 7,7\% ( $\mathrm{n}=1)$.

A avaliação psicológica foi realizada com sete gestantes. $\mathrm{Na}$ avaliação da ansiedade, verificou-se que 5 gestantes $(71,4 \%)$ apresentavam nível moderado; 1 gestante $(14,3 \%)$ se apresentou em nível mínimo, e 1, em nível leve $(14,3 \%)$. Na avaliação do estresse, verificou-se que 5 gestantes $(71,4 \%)$ se encontravam na fase de resistência, e $2(28,6 \%)$, na fase extrema de exaustão. Lembrando que nenhuma se mostrou nas fases 1 e 3 , alerta e quase exaustão, respectivamente.

Em relação às estratégias de enfrentamento (coping), observou-se que 3 gestantes $(42,8 \%)$ tiveram prevalência do enfrentamento "focado em práticas religiosas/pensamento fantasioso"; outras 3 gestantes buscaram suporte social para lidar com o estresse (42,8\%); e 1 (14,3\%) utilizou estratégias de enfrentamento "focadas no problema". Nenhuma gestante relatou estratégias de enfrentamento "focada na emoção".

Investigando a relação entre tipos de estratégias de coping, as fases do estresse e a prática de picamalácia, foi feita uma análise intragrupo quando se observou grande variabilidade dos dados. Das três gestantes que apresentaram estratégias "focadas em práticas religiosas", foi observada a ingestão de fruta verde com sal como prática de picamalácia, sendo que uma das gestantes ainda relatou comer pé de moleque com pão. Desse grupo, apenas uma gestante estava na fase de exaustão do estresse, as outras duas se encontravam na fase de resistência do estresse.

Relatado por outras três gestantes, estratégias de "busca por suporte social" pareceu se relacionar com diferentes tipos de picamalácia (tijolo/barro molhado, detergente de coco e naftalina e gelo), sendo que apenas uma gestante estava na fase de exaustão do estresse e as outras duas apresentaram sinais de estresse na fase de resistência. Já o coping "focalizado no problema" foi adotado por uma única gestante, que, na fase de resistência do estresse, relatou como prática de picamalácia ingerir frutas verdes com sal. Por fim, cabe destacar que das duas gestantes com coping "focando na busca por práticas religiosas" e da outra com coping "focado no problema", os tipos de picamalácia foram, respectivamente, manga com sal e tijolo/barro molhado.

Constatou-se grande variação intragrupo na análise de associações entre fase do estresse, tipo de picamalácia e tipo de coping, já que até mesmo gestantes classificadas em uma mesma fase de estresse apresentaram variadas práticas de picamalácia, assim como diferentes estratégias de enfrentamento.

\section{Discussão}

$\mathrm{Na}$ literatura são escassos os achados acerca do tema picamalácia e fatores nutricionais e psicológicos, especialmente durante a gestação. Muitos estudos supõem que a picamalácia esteja relacionada com questões psicológicas, como o estresse; porém, poucos discutem tais questões como possíveis causas ou desencadeadores da picamalácia $^{5,10,19}$. Em contrapartida, nossos dados confirmam que a picamalácia é apresentada como principal justificativa para o alívio de sintomatologia digestiva e para o alívio da ansiedade e do estresse presentes na gravidez.

Achados semelhantes são descritos com gestantes americanas, cuja ingestão de substâncias como argila, amido de milho e bicarbonato de sódio foi associada ao alívio da sintomatologia digestiva ${ }^{8,17}$. Igualmente, a picamalácia aparece como fator de proteção do estresse desencadeado na gestação ${ }^{5,10}$. Assim, a picamalácia pode 
ser um comportamento de adaptação, de aceitação, de representação de uma necessidade fisiológica ou de um desejo por uma substância não nutritiva como forma de alívio do desconforto e da ansiedade típicos da gestação.

Estudos sugerem a associação entre a picamalácia e a inadequação dietética, sendo descrita dentre mulheres que praticavam a picamalácia em comparação com as não praticantes; ingestões inferiores de carboidratos, proteínas, ferro heme e zinco, podendo causar baixo ganho de peso e deficiências nutricionais ${ }^{18}$. Com isso, ressalta-se a importância do diagnóstico precoce do transtorno na gestação, para tratá-lo ou preveni-lo, com orientação de alimentação equilibrada e uso de suplementos durante a gestação.

As principais substâncias não alimentares relatadas pelas gestantes foram: gelo, fruta verde com sal, tijolo/barro molhado e combinações atípicas de alimentos. Em estudo de revisão ${ }^{8}$ encontram-se achados semelhantes, nos quais a maior parte da informação sobre tal prática provém de estudos realizados nos Estados Unidos, especialmente na Califórnia, sendo as substâncias mais utilizadas gelo (pagofagia) e o amido de milho (amilofagia) ${ }^{8}$. Em estudo brasileiro verificou-se que o tijolo foi a substância mais consumida pelas gestantes $(20 \% \text { da amostra })^{26}$.

Em estudo prospectivo e descritivo foi verificado que a prevalência de picamalácia foi de 38\%, dentre 128 mulheres grávidas, sendo mais frequente entre gestantes de origem africana e com baixo peso pré-gestacional ${ }^{27}$.

A associação entre picamalácia e anemia foi verificada no presente estudo. Achados semelhantes são descritos em estudo longitudinal, no qual a picamalácia iniciada em períodos iniciais da gestação associou-se com anemia e redução significativa da concentração de hemoglobina no terceiro trimestre gestacional ${ }^{16}$. A anemia é uma intercorrência comum: estima-se uma prevalência de $50 \%$ dentre as gestantes brasileiras ${ }^{22}$, o que justifica a importância da investigação dessa prática ao longo da gestação e durante todas as consultas do pré-natal, a fim de prevenir e controlar a anemia gestacional.

Os achados do presente estudo sugerem uma possível associação entre intercorrências gestacionais e picamalácia, pois a maioria das gestantes estudadas apresentou alguma comorbidade, em conformidade com estudos que demonstram que gestantes que declaram prática de picamalácia apresentam maior chance de desenvolver hipertensão arterial, hipocalcemia e alterações hidroeletrolíticas, ainda que corrigidas quando os hábitos alimentares são $\operatorname{modificados}^{18}$. O fato de a picamalácia se associar à anemia gestacional e à presença de intercorrências gestacionais reforça a necessidade de investigação dessa prática no cenário da assistência pré-natal como um marcador de intercorrência gestacional.

Destaca-se ainda que $61,5 \%$ das gestantes estudadas que praticavam picamalácia também tinham $\mathrm{DM}$, sugerindo uma possível associação entre o DMG e o transtorno ${ }^{28}$. Essa possível associação é descrita em outro estudo ${ }^{18}$, no qual se sugere que gestantes com DM (prévio ou gestacional) teriam maior probabilidade de desenvolver a picamalácia e, com isso, maior chance de resultado perinatal indesejável.

A maioria das gestantes avaliadas psicologicamente apresentou indicadores de ansiedade em nível moderado, corroborando a literatura na área, que indica existir certo grau de ansiedade em toda a gestação devido ao reconhecimento dos riscos inerentes à própria gravidez ${ }^{11,12}$. Igualmente, todas as gestantes apresentaram quadro clínico de estresse, inclusive cinco delas na fase de resistência e duas na fase de exaustão do estresse. Tal dado não pode ser subestimado, uma vez que essas são as fases mais críticas do estresse, quando ele pode se tornar crônico, dificultando que a gestante consiga se adaptar e gerando distúrbios físicos graves e até psicológicos, como ansiedade e depressão ${ }^{29}$.

Igualmente, estes dados sugerem ser a picamalácia uma forma de a gestante lidar com os estressores próprios do ciclo gravídico-puerperal ${ }^{5,10,11}$, confirmados pelos poucos estudos existentes ${ }^{5,19,30}$. Dessa forma, a mulher tenta aliviar a tensão decorrente da ansiedade típica da gravidez, já que, segundo Vieira e Parizotto ${ }^{11}$, é um momento da vida dela caracterizado por diversas alterações emocionais, físicas e biopsicossociais, que podem, inclusive, deflagrar um período de luto, com exigência de elaboração psíquica dessa nova situação de vida. E, ainda, a necessidade de controle da alimentação durante a gestação também pode causar irritabilidade e ansiedade na gestante, podendo a picamalácia ser entendida como um mecanismo que diminuiria tal sentimento ${ }^{5,19}$.

Como já falado, a picamalácia pode ser uma resposta de proteção do indivíduo ao estresse psicológico, ou seja, um "mediador do estresse" agindo por meio do sistema imunológico, como o hábito nervoso análogo de roer as unhas, por exemplo ${ }^{5}$. Porém, a pouca literatura sobre a relação entre picamalácia e estresse psicológico se centra em estudos de casos individuais ou estudos nos quais o estresse foi medido de forma não padronizada ${ }^{10,30}$.

Em relação ao coping, avaliado pela EMEP, foi observado que um dos tipos de coping mais utilizados pelas gestantes foi a "busca por suporte social". Estratégias de enfrentamento baseadas no suporte social se relacionam com a ideia de que o apoio, tanto familiar quanto socioeconômico, pode facilitar o enfrentamento desse período de mudanças e de risco perinatal. Outro tipo de enfrentamento frequente foi o "focado na busca por práticas religiosas/pensamentos fantasiosos", adotado por $42,8 \%$ da amostra. Segundo Folkman e Moskowitz ${ }^{15}$, esse tipo de coping pode ter duas funções, pois quando se centra na religião pode colaborar na resolução do problema, ou ao contrário, quando a religião é considerada responsável absoluta pelo desfecho do mesmo, nega-se o problema por intermédio do uso de pensamento fantasioso. Desse modo, quando o enfrentamento se foca na 
religião, a gestante aguarda por uma resolução do problema sem participar ativamente do processo, o que deve ser mais bem investigado em futuros estudos.

Vale ressaltar que pareceu haver relação entre fatores nutricionais e psicológicos, uma vez que as gestantes participantes deste estudo revelaram ainda, durante as consultas de nutrição, sentir "muito desejo" de comer substâncias não alimentares ("isopor" e "pneu"), sem ter, de fato, consumido, por "não terem coragem" e "medo" de fazer mal à sua saúde e à do concepto. Ainda que a prática da picamalácia não tenha se consumado nesses casos, o relato desse "desejo", por si só, pode ser indício de futura deficiência nutricional, ou de presença de indicadores de estresse ou ansiedade, identificados na avaliação psicológica. Tal hipótese, no entanto, merece ser mais bem investigada em futuros estudos, com amostra mais ampla e delineamento longitudinal que permitam estabelecer correlações estatísticas confiáveis, o que não foi possível devido às limitações amostrais.

Como limitação do estudo podemos apontar a possível subestimação dos casos de picamalácia, pela dificuldade de as gestantes admitirem a prática. Uma dificuldade adicional em estudar a picamalácia é que esse transtorno não se encaixa facilmente em uma categoria conceitual e os instrumentos de avaliação dietética e psicológica não investigam o transtorno de forma apropriada.

Diante dos resultados do presente estudo pode-se concluir que a gravidez implica naturalmente em maior vulnerabilidade e desorganização de padrões anteriores, modificações fisiológicas e estados emocionais peculiares, que contribuem para a ocorrência de ansiedade na mulher. Logo, fatores próprios da gravidez podem contribuir para o surgimento da picamalácia na gestação, e seus riscos dependerão da frequência da prática e da substância ingerida. Contudo, casos de picamalácia na gestação são ainda subestimados e pouco valorizados, já que é uma desordem com difícil diagnóstico e notificação ${ }^{3}$, devido ao constrangimento da mulher ao abordar o assunto e consequente vergonha e medo ${ }^{18,19}$.

Por fim, considerando a falta de padronização na investigação da picamalácia na assistência pré-natal relacionada à dificuldade dos profissionais de saúde em abordar o tema ${ }^{2,17}$, destaca-se como importante a implantação de instrumentos padronizados em serviços de pré-natal que possam investigar a picamalácia de forma apropriada, tanto do ponto de vista nutricional quanto psicológico. Além disso, é fundamental que profissionais de saúde sejam conscientizados sobre a importância do diagnóstico precoce desse transtorno, a fim de implementarem práticas preventivas para a saúde materno-infantil como favorecedor de desfechos perinatais positivos.

\section{Contribuições dos autores}

Concepção e desenho - Ana Carolina Ayeta, Ana Cristina Barros da Cunha e Cláudia Saunders; análise e interpretação dos dados - Ana Carolina Ayeta, Cláudia Saunders e Ana Cristina Barros da Cunha; escrita e revisão do manuscrito - Ana Carolina Ayeta, Cláudia Saunders, Ana Cristina Barros da Cunha e Sonaly Petronilho Heidelmann.

\section{Agradecimentos}

À Universidade Federal do Rio de Janeiro (UFRJ) e ao Governo Federal, pela Bolsa de Residência Multiprofissional, e ao Conselho Nacional de Desenvolvimento Científico e Tecnológico (CNPq), pela Bolsa de Produtividade (PQ).

\section{Referências}

1. Phipps A, Fels H, Burns MS, Gerstenberger SL. Lead poisoning due to geophagia: the consumption of miniature pottery. Open J Pediatrics. 2012;2:60-6.

2. Mills ME. Craving more than food: the implications of pica in pregnancy. Nurs Womens Health. 2007; 11 (3):266-73.

3. Bhatia MS, Kaur N. Pagophagia - a common but rarely reported form of pica. J Clin Diagn Res. 2014;8(1):195-6.

4. López LB, Marigual M, Martín N, Mallorga M, Villagrán E, Zadorozne $M E$, et al. Characteristics of pica practice during pregnancy in a sample of Argentine women. J Obstet Gynaecol. 2012;32(2):150-3.

5. Young SL. Pica in pregnancy: new ideas about an old condition. Annu Rev Nutr. 2010;30:403-22.

6. Lumish RA, Young SL, Lee S, Cooper E, Pressman E, Guillet R, et al. Gestational iron deficiency is associated with pica behaviors in adolescents. J Nutr. 2014;144(10):1533-9.
7. American Psychiatric Association. Diagnostic and statistical manual of mental disorders: DSM-IV-TR. 4th ed. Washington (DC): American Psychiatric Association; 2000. p. 95-6.

8. Toker H, Ozdemir H, Ozan F, Turgut M, Goze F, Sencan M, et al. Dramatic oral findings belonging to a pica patient: a case report. Int Dent J. 2009;59(1):26-30.

9. Nyaruhucha $\mathrm{CN}$. Food cravings, aversions and pica among pregnant women in Dares Salaam, Tanzania. Tanzan J Health Res. 2009;1 1(1):29-34

10. Dunker KL, Alvarenga MS, Alves VP. Transtornos alimentares e gestação: uma revisão. J Bras Psiquiatr. 2009;58(1):60-8.

11. Vieira BD, Parizotto AP. Alterações psicológicas decorrentes do período gravídico. Unoesc Ciênc - ACBS. 2013;4(1):79-90.

12. Sarmento R, Setúbal MR. Abordagem psicológica em obstetrícia: aspectos emocionais da gravidez, parto e puerpério. Rev Ciênc Méd (Campinas). 2003;12(3):261-8. 
13. Lazarus RS, Folkman S. Stress, appraisal, and coping. New York: Springer; 1984.

14. Carver CS, Connor-Smith J. Personality and coping. Annu Rev Psychol. 2010;61:679-704.

15. Folkman S, Moskowitz JT. Coping: piffalls and promise. Annu Rev Psychol. 2004;55:745-74.

16. Saunders C, Padilha PC, Della Líbera B, Nogueira JL, Oliveira LM, Astulla A. [Pica: epidemiology and association with pregnancy complications]. Rev Bras Ginecol Obstet. 2009;31(9):440-6. Portuguese.

17. Thihalolipavan S, Candalla BM, Ehrlich J. Examining pica in NYC pregnant women with elevated blood lead levels. Matern Child Health J. 2013;17(1):49-55.

18. Ezzeddin N, Zavoshy R, Noroozi M, Jahanihashemi H, Riseh SH. Prevalence and risk factors for pica during pregnancy in Tehran, Iran. Eat Weight Disord. 2015 Jul 5. [Epub ahead of print].

19. Kachani AT, Cordás TA. Da ópera-bufa ao caos nosológico: pica. Rev Psiquiatr Clín. 2009;36(4): 162-9.

20. National Academy of Science. Institute of Medicine. Weight gain during pregnancy: reexamining the guidelines. Washington (DC): National Academies Press; 2009.

21. Brasil. Ministério da Saúde. Protocolos do Sistema de Vigilância Alimentar e Nutricional - SISVAN na assistência à saúde. Brasília (DF): Ministério da Saúde; 2008.
22. Brasil. Ministério da Saúde. Secretaria de Atenção à Saúde. Departamento de Atenção Básica. Atenção ao pré-natal de baixo risco. Brasília (DF): Editora do Ministério da Saúde; 2012.

23. Cunha JA. Manual da versão em português das Escalas Beck. São Paulo: Casa do Psicólogo; 2001.

24. Lipp MN. Manual do inventário de sintomas de stress para adultos de Lipp (ISSL). São Paulo: Casa do Psicólogo; 2000.

25. Seidl EMF, Trócolli BT, Zannon CMLC. Análise fatorial de uma medida de estratégias de enfrentamento. Psicol Teor Pesqui. $2001 ; 17(3): 225-34$

26. Mikkelsen TB, Andersen AM, Olsen SF. Pica in pregnancy in a privileged population: myth or reality. Acta Obstet Gynecol Scand. 2006;85(10): 1265-6.

27. Corbett RW, Ryan C, Weinrich SP. Pica in pregnancy: does it affect pregnancy outcomes? MCN Am J Matern Child Nurs. $2003 ; 28(3): 183-9$.

28. Gangopadhyay R, Karoshi M, Keith L. Anemia and pregnancy: a link to maternal chronic diseases. Int J Gynaecol Obstet. 2011;115 Suppl 1:S11-5.

29. Rodrigues OM, Schiavo RA. [Stress in pregnancy and puerperium: a correlation with postpartum depression]. Rev Bras Ginecol Obstet. 2011 ; 33(9):252-7. Portuguese.

30. Fiestas-Teque L. Pica en anemia severa: a propósito de un caso. Rev Neuropsiquiatr. 2014;77(2):123-5. 